\title{
Role of ischemic preconditioning in cardioprotective mechanisms of mCRP deposited myocardium in a rat model
}

${ }^{1}$ Eun Na Kim, MD, Ph.D., ${ }^{2}$ Jae-Sung Choi, MD, Ph.D., ${ }^{1}$ Chong Jai Kim, MD, Ph.D., ${ }^{3}$ So Ra Kim, ${ }^{4}$ Ki-Bong Kim, MD, Ph.D., ${ }^{2}$ Se Jin Oh, MD, Ph.D.

${ }^{1}$ Department of Pathology, University of Ulsan College of Medicine, Asan Medical Center, Seoul, Republic of Korea

${ }^{2}$ Department of Thoracic and Cardiovascular Surgery, SMG-SNU Boramae Medical Center, Seoul National University College of Medicine, Seoul, Republic of Korea ${ }^{3}$ Asan Laboratory of Perinatal Science, University of Ulsan College of Medicine, Asan Medical Center, Seoul, Republic of Korea

${ }^{4}$ Department of Thoracic and Cardiovascular Surgery, Seoul National University Hospital, Seoul National University College of Medicine, Seoul, Republic of Korea 


\section{Corresponding Author:}

Se Jin Oh, MD, Ph.D.

Department of Thoracic and Cardiovascular Surgery, Seoul National University College of Medicine, SMG-SNU Boramae Medical Center, 20, Boramae-ro 5-gil, Dongjak-gu, Seoul 07061, Republic of Korea

e-mail: sejinoh3@gmail.com

Telephone number: +82-2-870-2295

Fax number: +82-2-831-0714

Short Title: Ischemic preconditioning in myocardium with mCRP deposition

Keywords: Ischemic preconditioning, ischemia-reperfusion injury, C-reactive protein, myocardial infarction

Word count: 2852 


\section{Abstract}

The deposition of monomeric C-reactive protein (mCRP) in the myocardium aggravates ischemia-reperfusion injury (IRI) and myocardial infarction. Ischemic preconditioning (IPC) is known to protect the myocardium against IRI. We evaluated the effects of IPC on mCRP-deposited myocardium due to IRI in a rat model. Myocardial IRI was produced by ligation of the coronary artery. Direct IPC was applied before IRI using multiple short direct occlusions of the coronary artery. CRP was infused intravenously after IRI. The study groups included the following: sham ( $n=3)$, IRI only $(n=5)$, IRI + CRP $(n=9)$, and IPC + IRI + CRP $(n=6)$ groups. The infarct area and area at risk were assessed using Evans blue and 2,3,5-triphenyltetrazolium chloride (2,3,5-TTC) staining. Additionally, mCRP immunostaining and interleukin (IL)-6 mRNA reverse transcriptase-polymerase chain reaction (RT-PCR) were performed. In the IRI+CRP group, the infarcted area, mCRP deposition, and IL-6 mRNA expression were higher than those in the IRI only group. However, in the IPC+IRI+CRP group, the infarction (20\% vs. $34 \% \mathrm{p}=0.085)$ and mCRP myocardial deposition $(21 \%$ vs. $44 \%, \mathrm{p}=0.026)$ were lower and IL-6 mRNA expression was higher than those in the IRI+CRP group (fold change, 407 vs. $326, p=0.808$ ), although this was not statistically significant. IPC has cardioprotective effects against myocardial damage caused by mCRP deposition. This protective effect is related to the increase in IL-6 mRNA expression. 


\section{Introduction}

Ischemic heart disease is one of the most important causes of global death, causing over 7.2 million deaths in the world annually[1]. In order to reduce the myocardial infarct size and prevent poor clinical outcomes, coronary artery reperfusion of the ischemic myocardium is performed using thrombolytic therapy, percutaneous coronary intervention, or coronary arterial bypass grafting. However, when the ischemic myocardium is reperfused, it can result in paradoxical harmful effects that damage the myocardium - this is called ischemic reperfusion injury (IRI)[2]. Approximately 50\% of the final size of myocardial infarction is due to IRI[1]. Subsequently, $10 \%$ of deaths and $25 \%$ of cardiac failure following acute myocardial infarction is due to IRI even after treatment with reperfusion of the ischemic heart[3]. Therefore, minimizing IRI is the most important strategy to salvage the myocardium after an ischemic event.

In 1986, Murry et al. proposed the concept of the cardioprotective role of ischemic preconditioning (IPC); multiple, brief, non-lethal ischemic episodes followed by short reperfusion before the main prolonged ischemic injury reduces the infarct size due to the development of resistance to IRI and inhibition of lethal reperfusion injury[4]. For decades, many researchers have studied the efficacy and mechanisms of IPC, and the myocardial protective effects of IPC have been demonstrated in animal studies $[5,6]$, in vivo human heart studies[7-9], and in vitro studies[10]. Ischemic preconditioning not only reduces the infarct size by increasing the resistance of isolated myocytes to hypoxic injury[11], but also reduces anginal pain, ST-segment elevation and lactate production[12], and reduces post-ischemic arrhythmia[13, 14]. IPC also slows 
metabolism[15] and helps in the recovery of cardiac function after an ischemic event[16].

There is emerging experimental evidence that the deposition of monomeric Creactive protein (mCRP) exacerbates the damage to the heart due to IRI $[17,18]$. CRP is an acute phase reactant protein mainly produced in the liver during systemic infections and inflammation[19]. The serum level of CRP is not only an important prognostic and predictive marker for various cardiovascular diseases[20] including the clinical outcomes, death, and heart failure following myocardial infarction[21] but CRP itself also acts as a cause of direct damage to the cardiovascular tissue[22, 23].

CRP in serum exists in a pentameric form (pCRP), and when it encounters a damaged cell membrane it undergoes structural changes from pCRP to mCRP[24]. Subsequently, mCRP is deposited in the damaged tissue, thereby, activating the reactive oxygen species[25] and complement system[26]. This aggravates the inflammatory process and exacerbates myocardial damage[18]. Using a rat IRI model, we previously confirmed that if the serum CRP level is high during the myocardial ischemic-reperfusion insult, serum CRP is deposited in the myocardium as mCRP and the size of the myocardial infarction is increased[17]. Additionally, our previous work demonstrated that the microRNA profile of the myocardial area at risk changed drastically when CRP was high during the ischemic-reperfusion injury[27].

However, the role of IPC in the myocardium damaged by IRI with mCRP deposition has not been investigated. Therefore, in this study, we investigated whether IPC is protective against mCRP-induced myocardial damage in IRI settings using a rat acute myocardial IRI model. 


\section{Materials and methods}

\section{Animals}

We set up a myocardial IRI model using female Sprague-Dawley rats weighing between 220 and $270 \mathrm{~g}$ with a gestational age of $10-14$ weeks. The animals were treated according to the Guide for the Care and Use of Laboratory Animals (National Academy of Sciences, Washington, DC, USA). The protocols for animal use were approved by the Institutional Animal Care and Use Committee (IACUC) at the SMG-SNU Boramae Medical Center Biomedical Research Institute (approval number: 2016-0027). Anesthesia was administered with inhalation of isoflurane (4\%) for induction, followed by intraperitoneal administration of Tiletamine $\mathrm{HCl}$ and Zolazepam HCL (Zoletil $50^{\circledR}$, $0.12 \mathrm{~mL}$, Virbac, Carros, France) and xylazine (Rompun ${ }^{\circledR}, 2 \%, 0.02 \mathrm{~mL}$, Bayer Healthcare, Loos, France) for maintenance.

The rats were intubated with 16-gauge intravenous catheters (REF 382457; BD Medical, Sandy, Utah, USA) and connected to ventilators (683 rodent ventilator; Harvard Apparatus, Holliston, Massachusetts, USA). Positive pressure ventilation at room air with a tidal volume of $2.5 \mathrm{ml}(10 \mathrm{~mL} / \mathrm{kg}, 60 \mathrm{breath} / \mathrm{min})$ was used to prevent atelectasis during the procedure. We approached the heart through left thoracotomy via the fourth intercostal space. The pericardium was opened to expose the left coronary artery. Myocardial ischemic injury was produced by ligating the left anterior descending coronary artery (LAD) approximately $2 \mathrm{~mm}$ distal to the origin of LAD using 6-0 nylon double sutures, buttressed with a small piece of plastic tube (Fig 1). After 45 minutes of ischemia, we loosened the sutures to allow for reperfusion for 45 minutes. The pericardium was left open to expose LAD during the procedure. 
Fig 1. Ligation of the left anterior descending coronary artery, buttressed with a plastic tube.

\section{Experimental protocols}

The experimental protocols are illustrated in Fig 2. In the sham group $(\mathrm{n}=3)$, thoracotomy and pericardiotomy were performed, and the pericardium was opened and maintained for 90 minutes without any manipulation. Subsequently, the rats were euthanized and autopsy was performed (Fig 2A). In the group with IRI only (IRI only group, $\mathrm{n}=5$ ), the myocardium was excised quickly after 45 minutes of LAD ligation and 45 minutes of reperfusion (Fig 2B). In the group treated with CRP following IRI (IRI+CRP group, $n=9)$, high-purity ( $>99 \%$ ) human CRP obtained from human plasma (C4063; Sigma-Aldrich, Saint Louis, Missouri) was infused via the femoral vein after 45 minutes of ischemia with LAD ligation, just before reperfusion (Fig 2C). In the group treated with ischemic preconditioning (IPC) followed by IRI and CRP injection (IPC+IRI+CRP group, n=6), IPC was applied before LAD ligation; IPC included three occlusions for 3 minutes each followed by a 5-minute period of reperfusion after each occlusion (Fig 2D).

Fig 2. The experimental protocols.

\section{Evans blue and TTC staining to determine infarct area and}

\section{AAR}


To evaluate the non-ischemic area, ischemic but not infarcted viable area (area at risk, AAR), and infarcted area, we performed staining with Evans blue and 2,3,5triphenyltetrazolium chloride (TTC, Sigma Chemical, Saint Louis, MO). After euthanasia and before the removal of the heart, $1 \mathrm{~mL}$ diluted heparin solution (2500 IU heparin $/ \mathrm{mL}$ ) was infused via the coronary ostia after clamping the ascending aorta. We re-sutured the LAD artery using 6-0 nylon, and injected 1\% Evans blue solution to stain the perfused non-ischemic myocardium. Both AAR and infarcted area (whole ischemic area) do not stain with Evans blue solution[28]. After Evans blue perfusion, the heart was cut into four transverse sections at regular intervals from the apex to the base[29]. One of the middle sections was used to measure the ischemia and infarct size. This midportion was sliced again in 4-mm slices and one slice was incubated with TTC dissolved in $100 \mathrm{mmol} / \mathrm{L}$ of phosphate buffer for 15 minutes. With TTC staining, the viable area of the myocardium — the non-ischemic area — and the viable area at risk are stained deep red and the infarcted zone remains unstained and is, therefore, white. Therefore, double staining with Evans blue and TTC stains the infracted area white, AAR deep red, and non-ischemic area blue[30].

\section{Histopathologic analysis and Immunohistochemistry}

The middle section of the excised heart was fixed in $10 \%$ buffered formalin and embedded in paraffin. The $4-\mu \mathrm{m}$ tissue sections were stained using hematoxylin and eosin (H\&E), and immunostained with human monoclonal anti-CRP antibody (C1688; Sigma-Aldrich, Saint Louis, MO, USA; 1:400 dilution) which specifically detect the 24kD monomeric CRP epitope[31, 32]. OptiView DAB immunohistochemical detection 
kit (Roche Diagnostics, Mannheim, Germany) and a Benchmark XT autoimmunostainer (Ventana Medical Systems, Tucson, AZ, USA) were used for immunostaining. Thorough histopathologic examination with microscopy was performed by one pathologist (E.N.K).

\section{Image analysis}

Images of the heart specimens stained with Evans blue and TTC were captured with a digital camera DP26 (Olympus, Tokyo, Japan). Digital images of mCRP immunostaining were acquired using the Vectra automated imaging system (PerkinElmer, Waltham, MA, USA). The areas of the infarcted myocardium (white zone) and AAR (red zone) were automatically calculated with inForm (PerkinElmer) imaging analysis software. The size of the infarct was expressed as a percentage of the infarct area and the whole ischemic area (infarct / [infarct + AAR $] \times 100)$. mCRP immunolabeling was examined to determine the tissue distribution within each section. mCRP immunopositivity were expressed as the percentage of the mCRPimmunopositive area and the whole ischemic area (mCRP-immunostained area / [infarct $+\mathrm{AAR}] \times 100)$ as previously reported[17].

\section{Analysis of IL-6 mRNA expression assay in rat myocardium}

RNA was prepared using a miRNeasy Mini Kit (Qiagen, Hilden, Germany) according to the manufacturer's instructions. The extracted RNA $(1 \mu \mathrm{g})$ was reverse-transcribed using Reverse Transcription System (Promega, Madison, Wisconsin, USA), and cDNAs were amplified using GeneAmp PCR System 9700 (Applied Biosystems, Foster City, 
CA, USA). Quantitative reverse transcriptase-polymerase chain reaction (qRT-PCR) analysis of interleukin (IL)-6 was performed using TaqMan Gene Expression Assays (Rn01410330_m1; Applied Biosystems, Foster City, CA, USA) and 7900HT Fast RealTime PCR System (Applied Biosystems). The rat ACTB (Rn00667869_m1; Applied Biosystems, Foster City, CA) endogenous control was used for normalization.

\section{Statistical analysis}

Data was expressed as mean and standard deviation and plotted as mean with standard error of mean. To compare two groups, two-tailed $t$-test was used. A $P$-value $<0.05$ was considered statistically significant. Data analyses were performed using GraphPad Prism v5.0 (GraphPad Software, San Diego, CA, USA).

\section{Results}

\section{Areas of infarcted and ischemic myocardium}

After Evans blue and TTC staining, the non-ischemic area was stained blue, AAR was stained red, and infarct area was stained white, as depicted in Fig 3. As with our previous study[17], the areas of myocardium damaged by IRI demonstrated cellular changes along with contraction bands with intensely eosinophilic intracellular stripes on H\&E staining, thus confirming that IRI was performed well in the experiment[33].

Fig 3. Evans blue and 2,3,5-triphenyltetrazolium chloride (TTC) staining.
A. White area: infarcted area
B. Red area: area at risk (ischemic but not infarcted)
C. Blue area: non-ischemic area 
The percentage of infarct area and whole ischemic area (infarct / [infarct + AAR] $\times$ $100)$ tended to be higher in the IRI+CRP group than that in the IRI only group $(34 \pm 15 \%$ vs. $23 \pm 7 \%, p=0.116)$. After ischemic preconditioning, the size of the infarcted area decreased more in the IPC $+\mathrm{IRI}+\mathrm{CRP}$ group than that in the IRI+CRP group, although statistical significance was not reached $(21 \pm 7 \%$ vs. $34 \pm 15 \%, p=0.085$, Fig 4 A, 5$)$.

Fig 4. (A) The sham group without staining. TTC and Evans blue staining in the IRI only, IRI+CRP, and IPC+IRI+CRP groups. (B) mCRP immunohistochemistry in the sham, IRI only, IRI+CRP, and IPC+IRI+CRP groups.

mCRP, monomeric C-reactive protein; IRI, ischemia reperfusion injury; TTC, 2,3,5triphenyltetrazolium chloride.

Fig 5. The ratio of infarct area and whole ischemic area.

\section{mCRP immunohistochemistry}

While mCRP staining was faint and non-specific in the sham and IRI only groups, floating serum CRP was strongly and diffusely deposited on the damaged myocardium in the IRI+CRP group, not only in infarcted area, but also the viable AAR. This finding is consistent with our previous results[17](Fig 4B).

However, after applying direct IPC, the area of mCRP deposition in the ischemic myocardium (mCRP-immunostained area / [infarct + AAR] × 100)) was significantly lower than that in the IRI+CRP group $(21 \pm 16 \%$ vs. $44 \pm 19 \%, p=0.026$, Fig $4 B, 6)$. 
Fig 6. The ratio of mCRP-immunopositive area and whole ischemic area.

mCRP, monomeric C-reactive protein.

\section{IL-6 mRNA expression increased after CRP injection and}

\section{IPC}

The IL-6 mRNA expression level was highest in the IPC+IRI+CRP group, followed by the IRI+CRP and IRI only groups (fold change, IPC+IRI+CRP group, $408 \pm 273$,

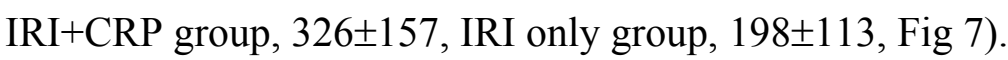

Fig 7. IL-6 mRNA expression.

IL, interleukin; mRNA, messenger RNA. 


\section{Discussion}

To our knowledge, this is the first report of the cardioprotective effect of IPC on mCRP deposited ischemic myocardium. IPC-induced cardioprotective effects are known to be achieved by various triggers, receptors, and mediators[34]. Especially, adenosine, opioid receptors[35], bradykinin[36], and free radicals[37] play important roles in IPC. In a heart preconditioned with IPC, lactate accumulates slowly, which results in antiinfarct effects[15]. IPC reduces intracellular acidification and affects the sodium-proton exchanger, cytoskeleton changes, and down regulation of TNF alpha[34]. However, despite numerous studies over a long period of time, the comprehensive mechanism of IPC is still controversial and much remains unclear[4, 38, 39]. And, there have been no studies focused on the effects of IPC on mCRP-deposited ischemic and damaged myocardium; therefore, we investigated whether IPC affects the amount of mCRP deposition and myocardial infarction in IRI settings. In this study, we found that shortterm direct IPC prior to IRI and CRP-infusion reduces mCRP deposition in the myocardium and infarction size and increases IL-6 mRNA expression.

Previous works have documented that mCRP deposition aggravates IRI-induced myocardial infarction. Thiele et al. reported that in a rat model of IRI, mCRP was localized to the infarcted myocardium and aggravated inflammation via phospholipase A2-dependent dissociation of circulating pCRP to mCRP[18]. Pepys et al. reported that they used a 1,6-bis(phosphocholine)-hexane that can bind and inhibit human CRP to reduce the size of myocardial infarction[40]. Additionally, we previously reported that mCRP was deposited not only in the 
infarcted area but also in the area at risk along with mitochondrial damage and complement activation[17].

IL-6 is a pleiotropic pro-inflammatory cytokine and one of the main factors for the stimulation of acute phase proteins, such as CRP[41]. IL-6 is known to be released from cardiomyocytes under hypoxic conditions, such as myocytes in the border zone of myocardial infarction, and IL-6 derived from hypoxic myocytes may play an important role in the aggravation of myocardial dysfunction following IRI[42, 43]. Therefore, IL6 was thought to result in hypertrophy and heart failure after ischemia by reducing the contractility of the myocardium[44]. In contrast, many studies have demonstrated that higher IL-6 levels during preconditioning play an organo-protective role[45-47]. Dawn et al. showed that ischemic preconditioning markedly upregulates IL-6 expression in the ischemic/reperfused myocardium. Furthermore, they demonstrated that IL-6 signaling plays an obligatory role in late preconditioning because IL-6 is required for the JAKSTAT signaling and upregulation of iNOS and COX-2, which are co-mediators of late preconditioning, and can, thus, aid in cardioprotection[45]. Similarly, Waldow et al. reported that if remote IPC was applied before IRI in a porcine lung, IL-6 increased more consistently than IRI alone, and the lung damage was alleviated[46]. In their in vivo study using ventricular cardiomyocytes from isolated rat hearts, Smart et al. demonstrated that IL-6-induced PI-3 kinase and NO-dependent protection of cardiomyocytes - associated with alterations in mitochondrial $\mathrm{Ca}^{2+}$ handling, inhibition of reperfusion-induced mitochondrial depolarization, swelling and loss of structural integrity, and suppression of cytosolic $\mathrm{Ca}^{2+}$ transients[47]. Additionally, in the present study, we confirmed that administering IPC before myocardial damage by IRI and 
mCRP deposition increases IL-6 mRNA expression and decreases the myocardial infarct size.

However, it is still controversial if increased IL-6 secretion plays an organo-protective role. In a porcine lung IRI model, Harkin et al. reported that IPC lowered IL-6 levels and resulted in a lung-protective effect[48]. The downstream pathways in which IL-6 plays a cardioprotective role are not well understood. Additionally, no detailed studies have been conducted to identify the specific signaling pathways when IL-6 is increased after mCRP deposition occurs. Further research is required to elucidate the mechanisms through which IL-6 acts on mCRP-deposited damaged tissue.

\section{Limitations}

In the above experiment, some observations that did not demonstrate statistical significance were observed. However, when CRP was deposited, the infarction area increased with the same trend as that reported in our previous work[17]. Animal studies with a larger sample size are required to further validate these findings. There is a possibility of disparity between the findings in animals and real-world clinical situations. So far, numerous attempts to prevent IRI that worked in animal experiments have actually been ineffective in clinical studies[1]. We used an ischemia model that directly occludes healthy coronary artery in young rats, which is very invasive for practical clinical applications. It is also virtually impossible to predict when profound ischemia will occur and apply preconditioning before IRI. Therefore, it may be difficult to create a useful clinical application from our experimental results. Furthermore, to 
elaborate the animal model to reflect the actual clinical situations, animal models with older animals and comorbidities, such as diabetes, hyperlipidemia, atherosclerosis, and hypertension should be used[1].

There are many clinical situations in which serum CRP can be elevated in cardiac ischemia. Baseline CRP levels are moderately elevated in obese persons or those who smoke or have diabetes or hypertension. Additionally, CRP levels increase dramatically in patients with myocardial infarction[49]. If a patient with a cardiovascular event has elevated serum CRP level for any reason, the serum CRP will deteriorate myocardial function while getting deposited in the damaged myocardium. We, therefore, can infer that in these clinical situations, IPC will function to protect the mCRP deposited myocardium. Additionally, our experiments provide clues to understand the mechanisms of aggravation of ischemia due to $\mathrm{mCRP}$ and the protective mechanisms of ischemic preconditioning. If the mechanism by which IPC protects the myocardium that has undergone mCRP deposition is understood completely, it will provide a basis for developing a preconditioning mimetic agent.

\section{Conclusion}

Ischemic preconditioning protects against myocardial damage caused by IRI and mCRP deposition. Additionally, this protective effect is believed to be associated with enhanced IL-6 mRNA expression.

\section{Acknowledgements}


We thank Kyung Min Park, Gil-Je Lee and Yun Jae Kim for the image analysis, and

Eun Jung Jeon for the molecular work. And we would like to thank Editage

(www.editage.co.kr) for English language editing.

\section{Financial support}

This work was funded by a clinical research grant-in-aid from the Seoul Metropolitan

Government Seoul National University (SMG-SNU) Boramae Medical Center (03-

2015-11). The funders had no role in study design, data collection and analysis, decision to publish, or preparation of the manuscript.

\section{Potential conflicts of interest}

The authors have declared that no competing interests exist. 


\section{References}

1. Yellon DM, Hausenloy DJ. Myocardial reperfusion injury. N Engl J Med. 2007;357(11):112135. Epub 2007/09/15. doi: 10.1056/NEJMra071667. PubMed PMID: 17855673.

2. Jennings RB, Sommers HM, Smyth GA, Flack HA, Linn H. Myocardial necrosis induced by temporary occlusion of a coronary artery in the dog. Arch Pathol. 1960;70:68-78. Epub 1960/07/01. PubMed PMID: 14407094.

3. Keeley EC, Boura JA, Grines CL. Primary angioplasty versus intravenous thrombolytic therapy for acute myocardial infarction: a quantitative review of 23 randomised trials. Lancet. 2003;361(9351):13-20. Epub 2003/01/09. doi: 10.1016/S0140-6736(03)12113-7. PubMed PMID: 12517460.

4. Murry CE, Jennings RB, Reimer KA. Preconditioning with ischemia: a delay of lethal cell injury in ischemic myocardium. Circulation. 1986;74(5):1124-36. Epub 1986/11/01. PubMed PMID: 3769170.

5. Liu Y, Downey JM. Ischemic preconditioning protects against infarction in rat heart. Am J Physiol. 1992;263(4 Pt 2):H1107-12. Epub 1992/10/01. doi: 10.1152/ajpheart.1992.263.4.H1107. PubMed PMID: 1415759.

6. Schultz JE, Rose E, Yao Z, Gross GJ. Evidence for involvement of opioid receptors in ischemic preconditioning in rat hearts. Am J Physiol. 1995;268(5 Pt 2):H2157-61. Epub 1995/05/01. doi: 10.1152/ajpheart.1995.268.5.H2157. PubMed PMID: 7771566.

7. Yellon DM, Alkhulaifi AM, Pugsley WB. Preconditioning the human myocardium. Lancet. 1993;342(8866):276-7. Epub 1993/07/31. doi: 10.1016/0140-6736(93)91819-8. PubMed PMID: 8101304.

8. Kloner RA, Yellon D. Does ischemic preconditioning occur in patients? J Am Coll Cardiol. 1994;24(4):1133-42. Epub 1994/10/01. doi: 10.1016/0735-1097(94)90880-x. PubMed PMID: 7930208. 9. Dana A, Sumeray MS, Yellon DM. Ischaemic preconditioning: a clinical perspective. Hosp Med. 1998;59(3):216-20. Epub 1998/08/29. PubMed PMID: 9722349. 
10. Ikonomidis JS, Tumiati LC, Weisel RD, Mickle DA, Li RK. Preconditioning human ventricular cardiomyocytes with brief periods of simulated ischaemia. Cardiovasc Res. 1994;28(8):1285-91. Epub 1994/08/01. doi: 10.1093/cvr/28.8.1285. PubMed PMID: 7954635.

11. Armstrong S, Ganote CE. Adenosine receptor specificity in preconditioning of isolated rabbit cardiomyocytes: evidence of A3 receptor involvement. Cardiovasc Res. 1994;28(7):1049-56. Epub 1994/07/01. doi: 10.1093/cvr/28.7.1049. PubMed PMID: 7525060.

12. Deutsch E, Berger M, Kussmaul WG, Hirshfeld JW, Jr., Herrmann HC, Laskey WK. Adaptation to ischemia during percutaneous transluminal coronary angioplasty. Clinical, hemodynamic, and metabolic features. Circulation. 1990;82(6):2044-51. Epub 1990/12/01. doi:

10.1161/01.cir.82.6.2044. PubMed PMID: 2242528.

13. Shiki K, Hearse DJ. Preconditioning of ischemic myocardium: reperfusion-induced arrhythmias. Am J Physiol. 1987;253(6 Pt 2):H1470-6. Epub 1987/12/01. doi:

10.1152/ajpheart.1987.253.6.H1470. PubMed PMID: 3425747.

14. Wu ZK, Iivainen T, Pehkonen E, Laurikka J, Tarkka MR. Ischemic preconditioning suppresses ventricular tachyarrhythmias after myocardial revascularization. Circulation. 2002;106(24):3091-6. Epub 2002/12/11. doi: 10.1161/01.cir.0000041430.32233.5b. PubMed PMID: 12473557.

15. Murry CE, Richard VJ, Reimer KA, Jennings RB. Ischemic preconditioning slows energy metabolism and delays ultrastructural damage during a sustained ischemic episode. Circ Res. 1990;66(4):913-31. Epub 1990/04/01. doi: 10.1161/01.res.66.4.913. PubMed PMID: 2317895.

16. Cave AC, Hearse DJ. Ischaemic preconditioning and contractile function: studies with normothermic and hypothermic global ischaemia. J Mol Cell Cardiol. 1992;24(10):1113-23. Epub 1992/10/01. doi: 10.1016/0022-2828(92)93176-k. PubMed PMID: 1479613.

17. Oh SJ, Na Kim E, Jai Kim C, Choi JS, Kim KB. The effect of C-reactive protein deposition on myocardium with ischaemia-reperfusion injury in rats. Interact Cardiovasc Thorac Surg. 2017;25(2):2607. Epub 2017/05/06. doi: 10.1093/icvts/ivx107. PubMed PMID: 28475685.

18. Thiele JR, Habersberger J, Braig D, Schmidt Y, Goerendt K, Maurer V, et al. Dissociation of pentameric to monomeric C-reactive protein localizes and aggravates inflammation: in vivo proof of a powerful proinflammatory mechanism and a new anti-inflammatory strategy. Circulation. 
2014;130(1):35-50. Epub 2014/07/02. doi: 10.1161/CIRCULATIONAHA.113.007124. PubMed PMID: 24982116.

19. Gabay C, Kushner I. Acute-phase proteins and other systemic responses to inflammation. N Engl J Med. 1999;340(6):448-54. Epub 1999/02/11. doi: 10.1056/NEJM199902113400607. PubMed PMID: 9971870.

20. Liuzzo G, Biasucci LM, Gallimore JR, Grillo RL, Rebuzzi AG, Pepys MB, et al. The prognostic value of C-reactive protein and serum amyloid a protein in severe unstable angina. $\mathrm{N}$ Engl J Med. 1994;331(7):417-24. Epub 1994/08/18. doi: 10.1056/NEJM199408183310701. PubMed PMID: 7880233.

21. Suleiman M, Khatib R, Agmon Y, Mahamid R, Boulos M, Kapeliovich M, et al. Early inflammation and risk of long-term development of heart failure and mortality in survivors of acute myocardial infarction predictive role of C-reactive protein. J Am Coll Cardiol. 2006;47(5):962-8. Epub 2006/03/07. doi: 10.1016/j.jacc.2005.10.055. PubMed PMID: 16516078.

22. Griselli M, Herbert J, Hutchinson WL, Taylor KM, Sohail M, Krausz T, et al. C-reactive protein and complement are important mediators of tissue damage in acute myocardial infarction. J Exp Med. 1999;190(12):1733-40. Epub 1999/12/22. doi: 10.1084/jem.190.12.1733. PubMed PMID: 10601349; PubMed Central PMCID: PMCPMC2195725.

23. Li JJ, Fang CH. C-reactive protein is not only an inflammatory marker but also a direct cause of cardiovascular diseases. Med Hypotheses. 2004;62(4):499-506. Epub 2004/03/31. doi:

10.1016/j.mehy.2003.12.014. PubMed PMID: 15050096.

24. Khreiss T, Jozsef L, Potempa LA, Filep JG. Loss of pentameric symmetry in C-reactive protein induces interleukin-8 secretion through peroxynitrite signaling in human neutrophils. Circ Res. 2005;97(7):690-7. Epub 2005/08/27. doi: 10.1161/01.RES.0000183881.11739.CB. PubMed PMID: 16123332.

25. Thiele JR, Zeller J, Kiefer J, Braig D, Kreuzaler S, Lenz Y, et al. A Conformational Change in C-Reactive Protein Enhances Leukocyte Recruitment and Reactive Oxygen Species Generation in Ischemia/Reperfusion Injury. Front Immunol. 2018;9:675. Epub 2018/05/02. doi: 10.3389/fimmu.2018.00675. PubMed PMID: 29713320; PubMed Central PMCID: PMCPMC5911593. 
26. Thompson D, Pepys MB, Wood SP. The physiological structure of human C-reactive protein and its complex with phosphocholine. Structure. 1999;7(2):169-77. Epub 1999/06/16. doi: 10.1016/S0969-2126(99)80023-9. PubMed PMID: 10368284.

27. Kim EN, Kim CJ, Kim SR, Song JA, Choe H, Kim KB, et al. High serum CRP influences myocardial miRNA profiles in ischemia-reperfusion injury of rat heart. PLoS One. 2019;14(5):e0216610. Epub 2019/05/08. doi: 10.1371/journal.pone.0216610. PubMed PMID: 31063484; PubMed Central PMCID: PMCPMC6504103.

28. Redfors B, Shao Y, Omerovic E. Myocardial infarct size and area at risk assessment in mice. Exp Clin Cardiol. 2012;17(4):268-72. PubMed PMID: 23592952; PubMed Central PMCID: PMCPMC3627291.

29. Pfeffer MA, Pfeffer JM, Fishbein MC, Fletcher PJ, Spadaro J, Kloner RA, et al. Myocardial infarct size and ventricular function in rats. Circ Res. 1979;44(4):503-12. PubMed PMID: 428047. 30. Rodrigues AC, Hataishi R, Ichinose F, Bloch KD, Derumeaux G, Picard MH, et al. Relationship of systolic dysfunction to area at risk and infarction size after ischemia-reperfusion in mice. J Am Soc Echocardiogr. 2004;17(9):948-53. doi: 10.1016/j.echo.2004.05.014. PubMed PMID: 15337959.

31. Schwedler SB, Guderian F, Dammrich J, Potempa LA, Wanner C. Tubular staining of modified C-reactive protein in diabetic chronic kidney disease. Nephrol Dial Transplant. 2003;18(11):2300-7. PubMed PMID: 14551357.

32. Slevin M, Matou-Nasri S, Turu M, Luque A, Rovira N, Badimon L, et al. Modified C-reactive protein is expressed by stroke neovessels and is a potent activator of angiogenesis in vitro. Brain Pathol. 2010;20(1):151-65. doi: 10.1111/j.1750-3639.2008.00256.x. PubMed PMID: 19170684.

33. Basso $\mathrm{C}$, Thiene $\mathrm{G}$. The pathophysiology of myocardial reperfusion: a pathologist's perspective. Heart. 2006;92(11):1559-62. doi: 10.1136/hrt.2005.086959. PubMed PMID: 16547203; PubMed Central PMCID: PMCPMC1861239.

34. Eisen A, Fisman EZ, Rubenfire M, Freimark D, McKechnie R, Tenenbaum A, et al. Ischemic preconditioning: nearly two decades of research. A comprehensive review. Atherosclerosis. 2004;172(2):201-10. Epub 2004/03/17. doi: 10.1016/S0021-9150(03)00238-7. PubMed PMID: 15019529. 
35. McCully JD, Uematsu M, Parker RA, Levitsky S. Adenosine-enhanced ischemic preconditioning provides enhanced cardioprotection in the aged heart. Ann Thorac Surg. 1998;66(6):2037-43. Epub 1999/02/04. doi: 10.1016/s0003-4975(98)01042-x. PubMed PMID: 9930490. 36. Wall TM, Sheehy R, Hartman JC. Role of bradykinin in myocardial preconditioning. J Pharmacol Exp Ther. 1994;270(2):681-9. Epub 1994/08/01. PubMed PMID: 8071859.

37. Baines CP, Goto M, Downey JM. Oxygen radicals released during ischemic preconditioning contribute to cardioprotection in the rabbit myocardium. J Mol Cell Cardiol. 1997;29(1):207-16. Epub 1997/01/01. doi: 10.1006/jmcc.1996.0265. PubMed PMID: 9040035.

38. Yellon DM, Baxter GF, Garcia-Dorado D, Heusch G, Sumeray MS. Ischaemic preconditioning: present position and future directions. Cardiovasc Res. 1998;37(1):21-33. Epub 1998/04/16. PubMed PMID: 9539854.

39. Garcia-Dorado D, Barba I, Inserte J. Twenty-five years of preconditioning: are we ready for ischaemia? From coronary occlusion to systems biology and back. Cardiovasc Res. 2011;91(3):378-81. Epub 2011/06/11. doi: 10.1093/cvr/cvr140. PubMed PMID: 21659485.

40. Pepys MB, Hirschfield GM, Tennent GA, Gallimore JR, Kahan MC, Bellotti V, et al. Targeting C-reactive protein for the treatment of cardiovascular disease. Nature. 2006;440(7088):1217-21. Epub 2006/04/28. doi: 10.1038/nature04672. PubMed PMID: 16642000.

41. Gauldie J, Richards C, Harnish D, Lansdorp P, Baumann H. Interferon beta 2/B-cell stimulatory factor type 2 shares identity with monocyte-derived hepatocyte-stimulating factor and regulates the major acute phase protein response in liver cells. Proc Natl Acad Sci U S A.

1987;84(20):7251-5. Epub 1987/10/01. doi: 10.1073/pnas.84.20.7251. PubMed PMID: 2444978; PubMed Central PMCID: PMCPMC299269.

42. Yamauchi-Takihara K, Ihara Y, Ogata A, Yoshizaki K, Azuma J, Kishimoto T. Hypoxic stress induces cardiac myocyte-derived interleukin-6. Circulation. 1995;91(5):1520-4. Epub 1995/03/01. doi: 10.1161/01.cir.91.5.1520. PubMed PMID: 7867193.

43. Gwechenberger M, Mendoza LH, Youker KA, Frangogiannis NG, Smith CW, Michael LH, et al. Cardiac myocytes produce interleukin-6 in culture and in viable border zone of reperfused infarctions. Circulation. 1999;99(4):546-51. Epub 1999/02/02. doi: 10.1161/01.cir.99.4.546. PubMed PMID: 9927402. 
44. Tsutamoto T, Hisanaga T, Wada A, Maeda K, Ohnishi M, Fukai D, et al. Interleukin-6 spillover in the peripheral circulation increases with the severity of heart failure, and the high plasma level of interleukin-6 is an important prognostic predictor in patients with congestive heart failure. J Am Coll Cardiol. 1998;31(2):391-8. Epub 1998/02/14. doi: 10.1016/s0735-1097(97)00494-4. PubMed PMID: 9462584.

45. Dawn B, Xuan YT, Guo Y, Rezazadeh A, Stein AB, Hunt G, et al. IL-6 plays an obligatory role in late preconditioning via JAK-STAT signaling and upregulation of iNOS and COX-2. Cardiovasc Res. 2004;64(1):61-71. Epub 2004/09/15. doi: 10.1016/j.cardiores.2004.05.011. PubMed PMID: 15364614; PubMed Central PMCID: PMCPMC3691700.

46. Waldow T, Alexiou K, Witt W, Albrecht S, Wagner F, Knaut M, et al. Protection against acute porcine lung ischemia/reperfusion injury by systemic preconditioning via hind limb ischemia. Transpl Int. 2005;18(2):198-205. Epub 2005/02/05. doi: 10.1111/j.1432-2277.2004.00005.x. PubMed PMID: 15691273.

47. Smart N, Mojet MH, Latchman DS, Marber MS, Duchen MR, Heads RJ. IL-6 induces PI 3kinase and nitric oxide-dependent protection and preserves mitochondrial function in cardiomyocytes. Cardiovasc Res. 2006;69(1):164-77. Epub 2005/10/13. doi: 10.1016/j.cardiores.2005.08.017. PubMed PMID: 16219301.

48. Harkin DW, Barros D'Sa AA, McCallion K, Hoper M, Campbell FC. Ischemic preconditioning before lower limb ischemia--reperfusion protects against acute lung injury. J Vasc Surg. 2002;35(6):1264-73. Epub 2002/06/04. doi: 10.1067/mva.2002.121981. PubMed PMID: 12042740. 49. Kitsis RN, Jialal I. Limiting myocardial damage during acute myocardial infarction by inhibiting C-reactive protein. N Engl J Med. 2006;355(5):513-5. Epub 2006/08/04. doi: 10.1056/NEJMcibr063197. PubMed PMID: 16885557. 


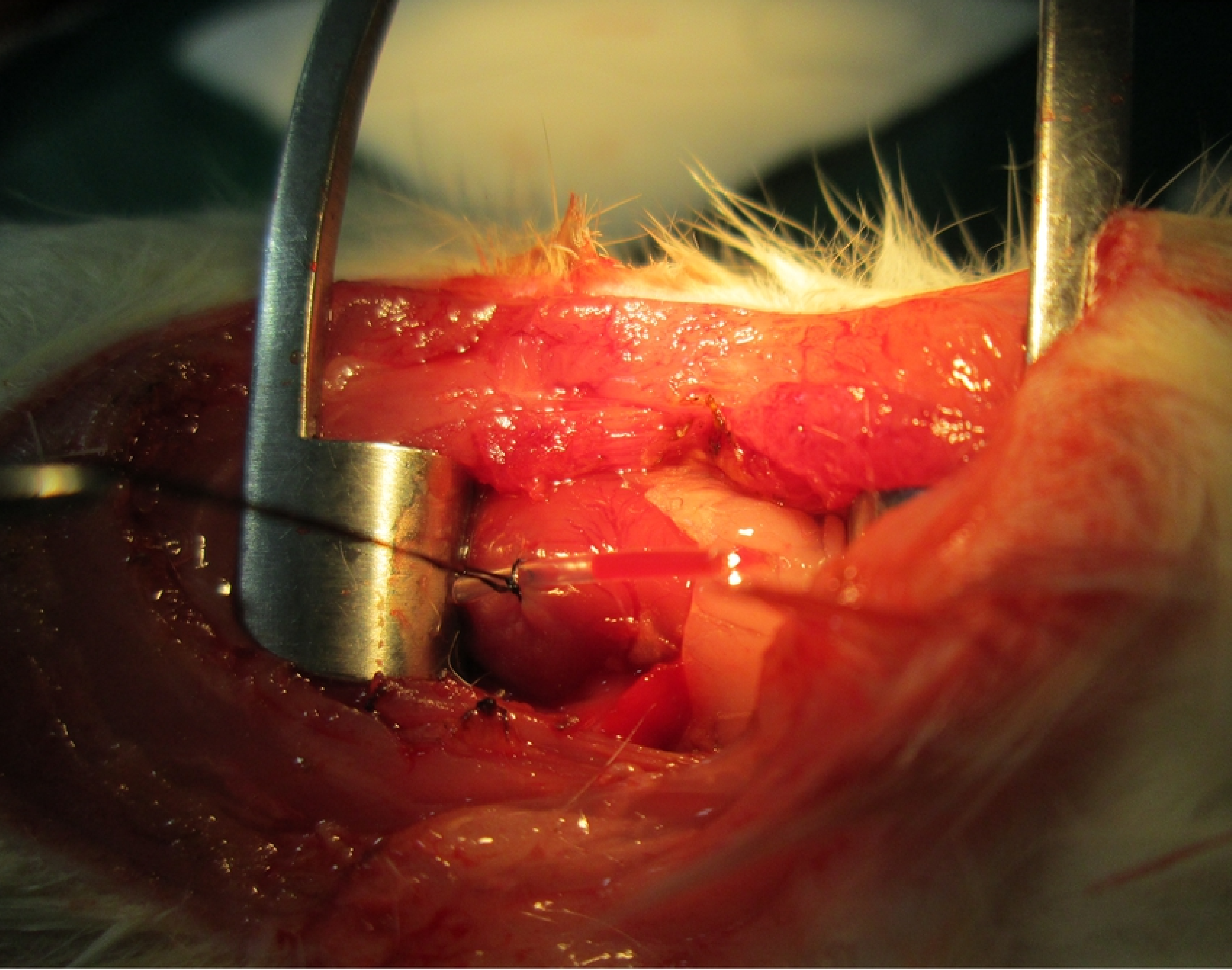

Fig 1 



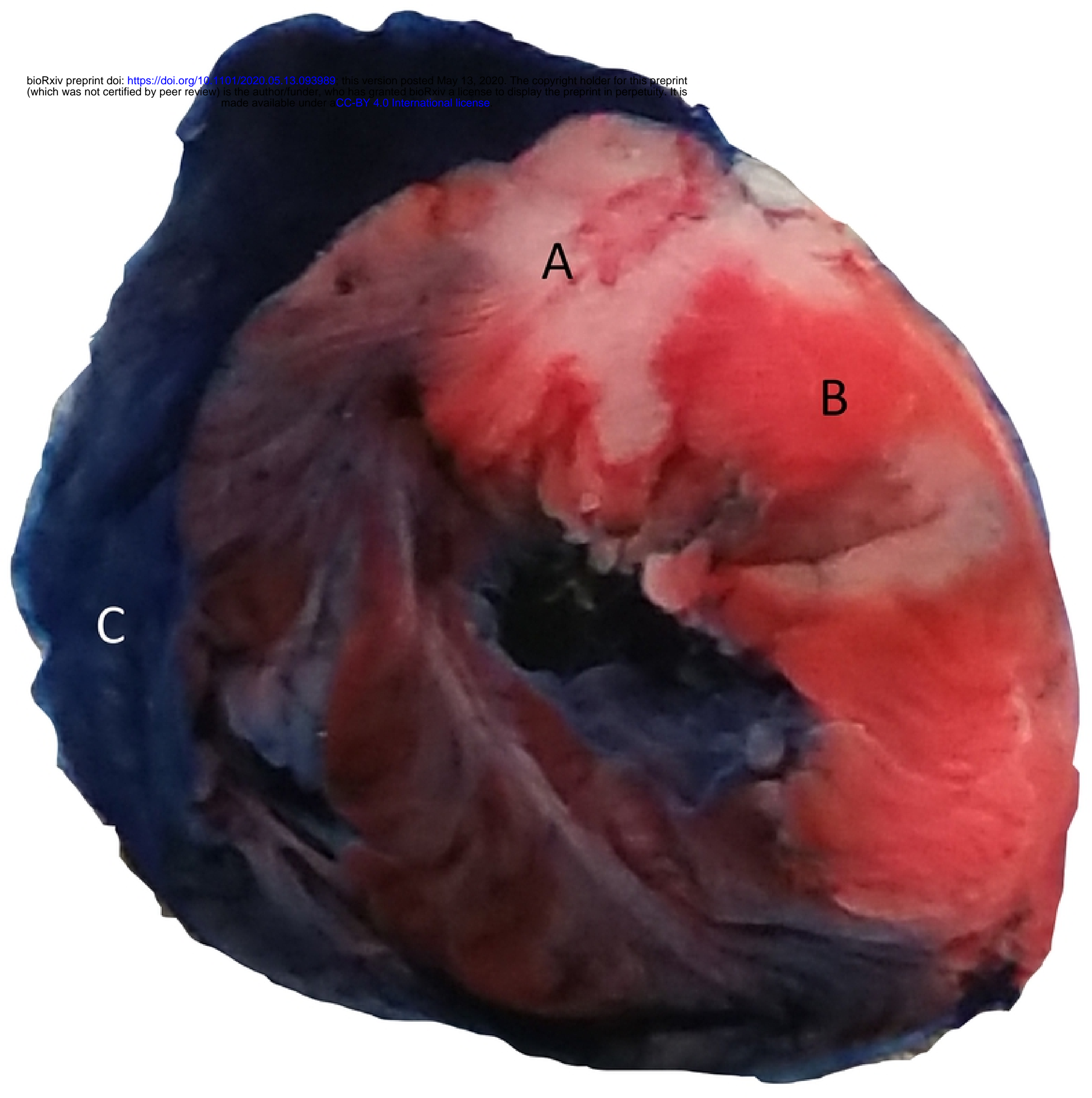

Fig 3 
A

Sham

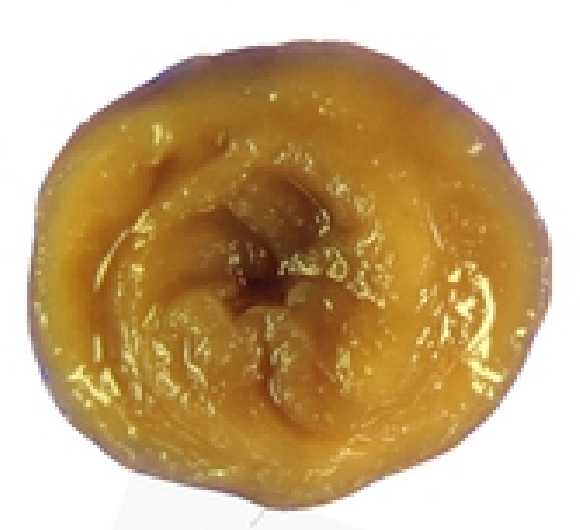

B
IRI only

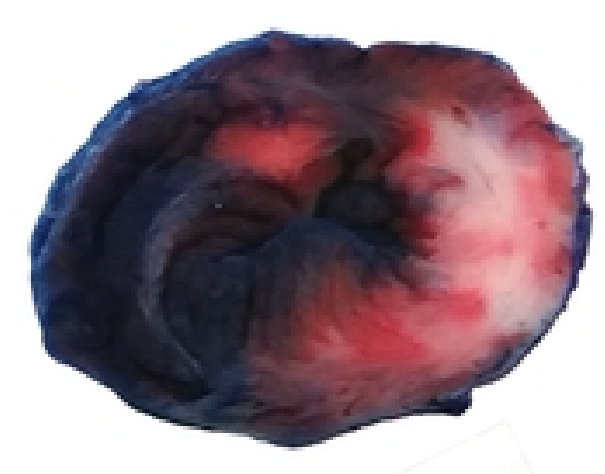

IRI+CRP

$|P C+| R \mid+C R P$
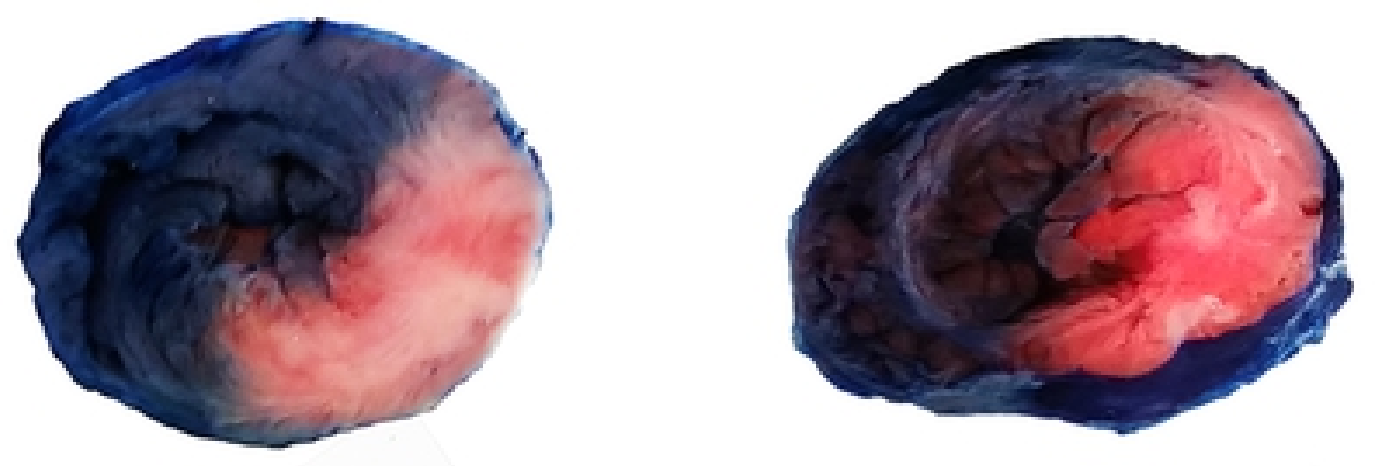

Fig 4 


\section{Infarct area/ Whole ischaemic area}

$\%$

80

biof xiv preprint doi: https://doi.org/10.1101/2020.05.13.093989; this version posted May 13, 2020. The copyright holder for this preprint biof xiv preprint doi: https://doi.org/10.1 is the author/funder, who has granted bioRxiv a license to display the preprint in perpetuity lt is

$p=0.116$

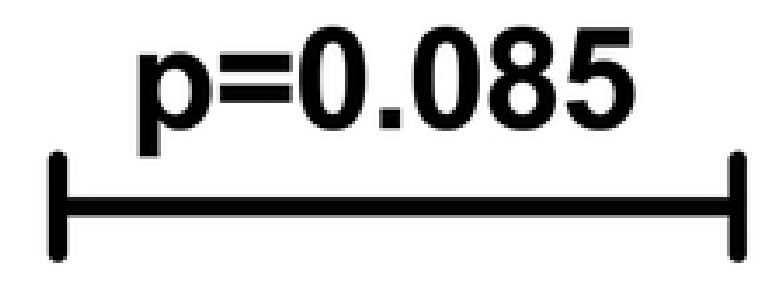

60
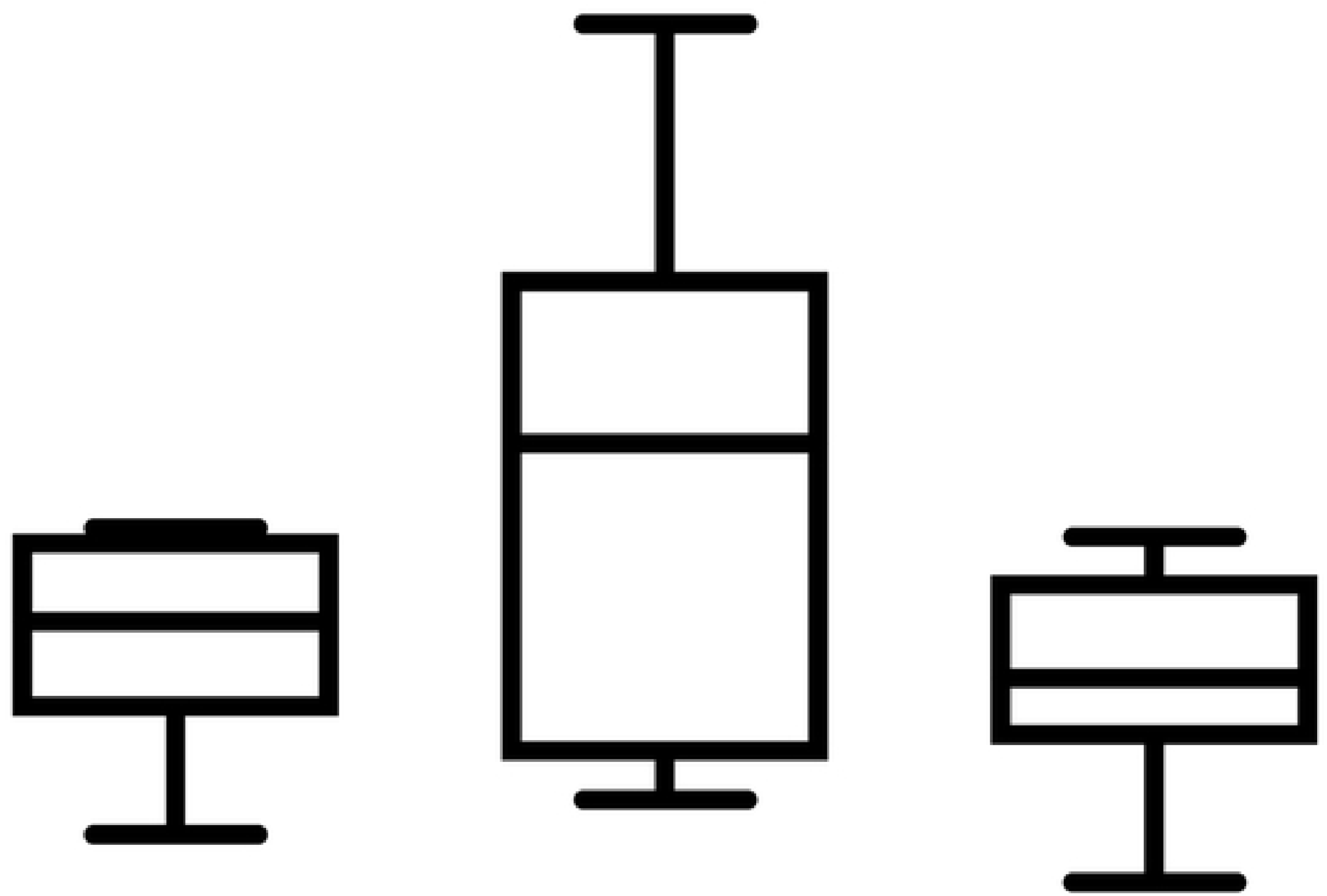

0
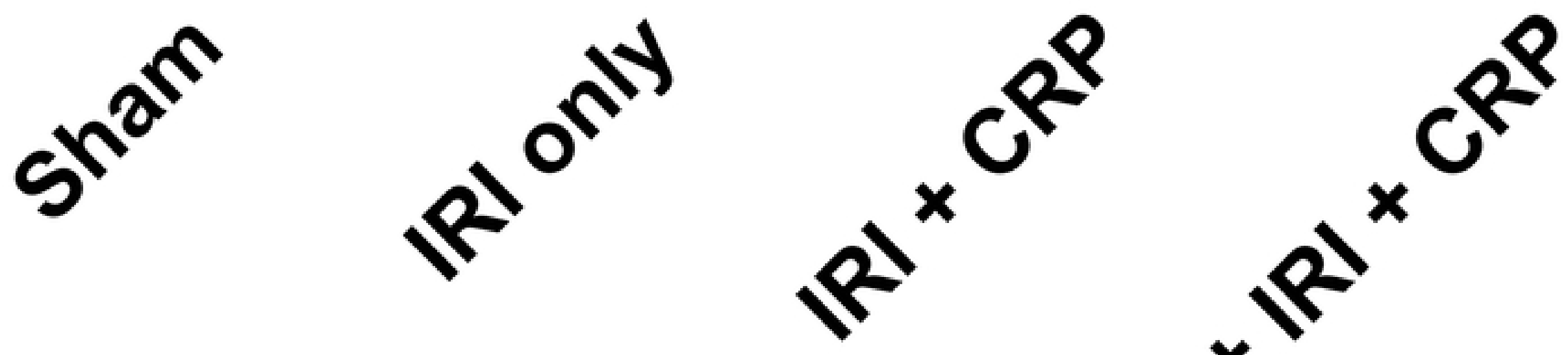

Fig 5 


\section{mCRP-immunopositive area/ whole ischaemic area}

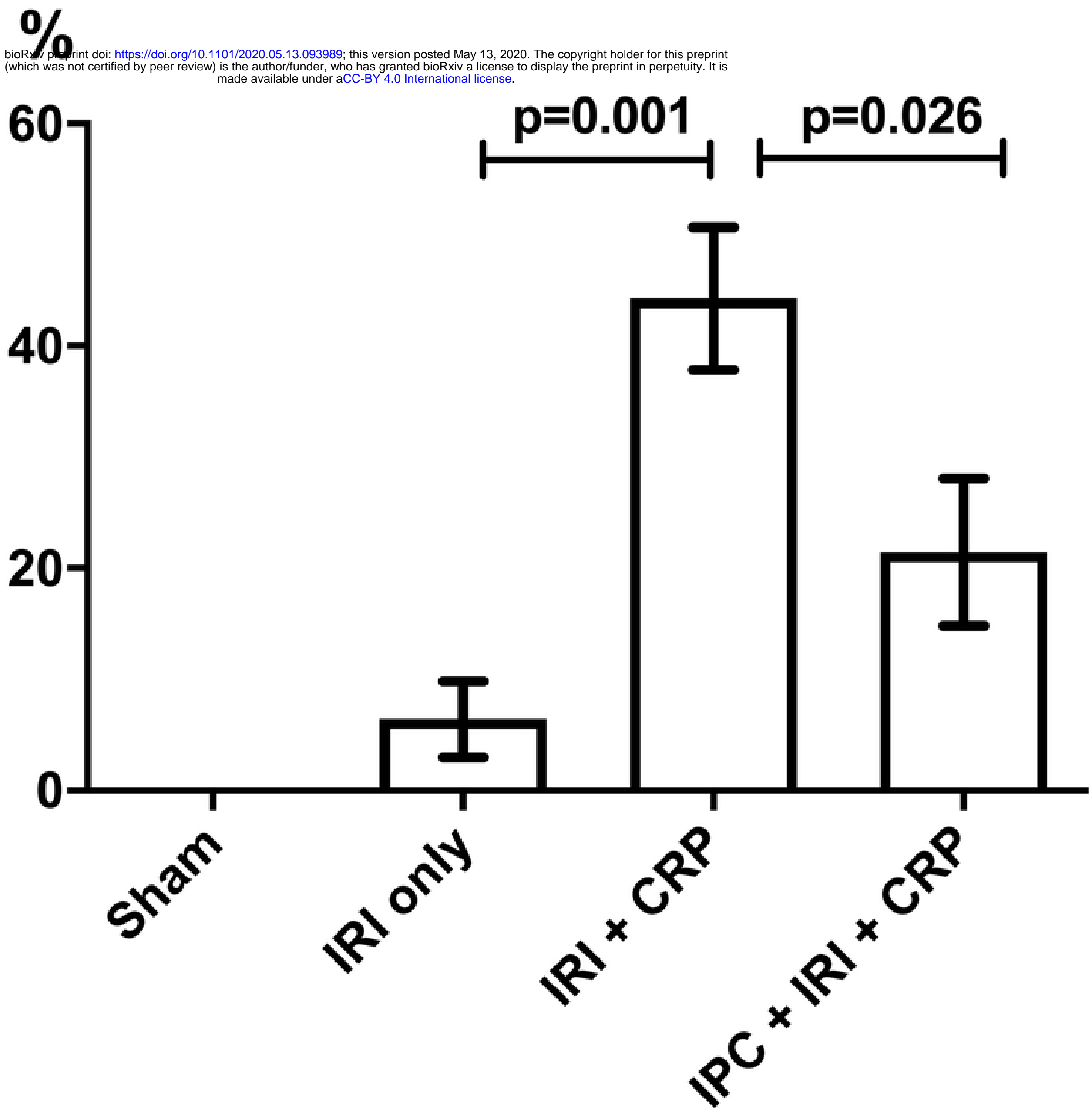

Fig 6 


\section{IL-6 mRNA expression}

bioRxiv preprint doi: https://doi.org/10.1101/2020.05.13.093989; this version posted May 13, 2020. The copyright holder for this preprint

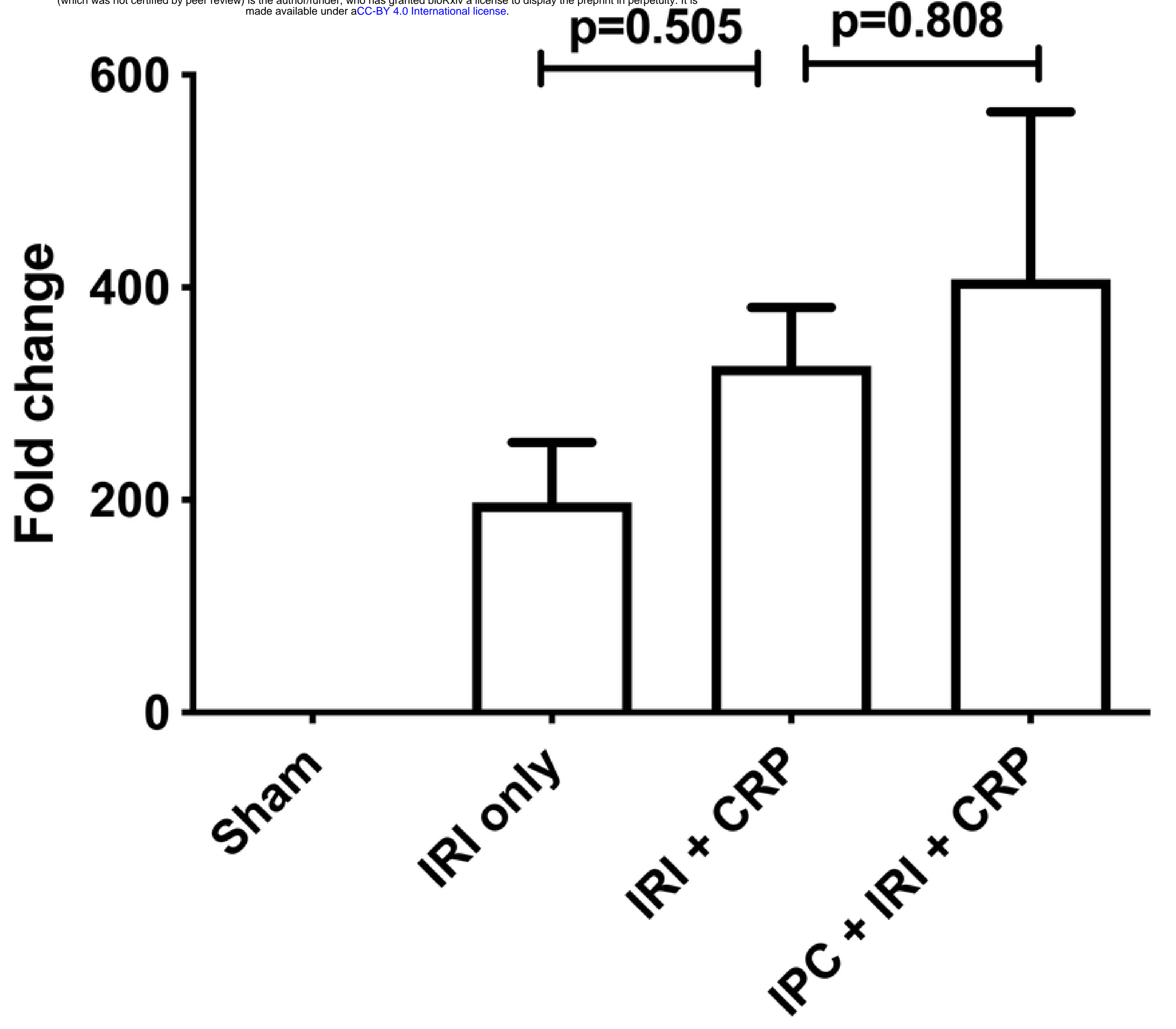

Fig 7 